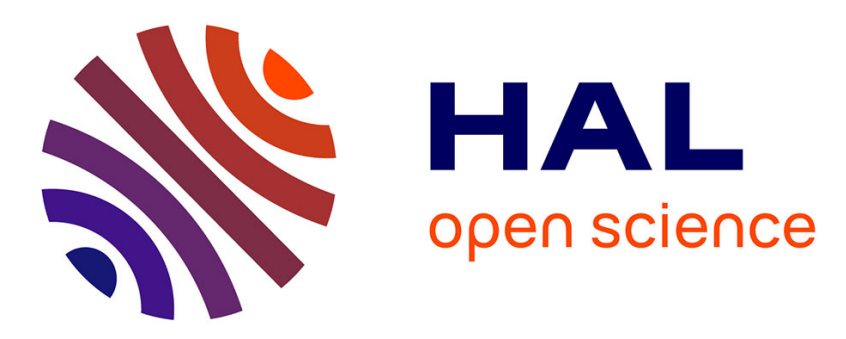

\title{
Caractérisation microstructurale 3D et densification locale d'isolants fibreux cellulosiques sollicités en compression
}

\author{
Christine Delisée, Eric Badel, Jérôme Lux, Jérôme J. Malvestio
}

\section{> To cite this version:}

Christine Delisée, Eric Badel, Jérôme Lux, Jérôme J. Malvestio. Caractérisation microstructurale 3D et densification locale d'isolants fibreux cellulosiques sollicités en compression. European Journal of Environmental and Civil Engineering, 2009, 13 (4), pp.429-442. 10.1080/19648189.2009.9693121. hal-00369709

\section{HAL Id: hal-00369709 \\ https://hal.science/hal-00369709}

Submitted on 5 Mar 2019

HAL is a multi-disciplinary open access archive for the deposit and dissemination of scientific research documents, whether they are published or not. The documents may come from teaching and research institutions in France or abroad, or from public or private research centers.
L'archive ouverte pluridisciplinaire HAL, est destinée au dépôt et à la diffusion de documents scientifiques de niveau recherche, publiés ou non, émanant des établissements d'enseignement et de recherche français ou étrangers, des laboratoires publics ou privés. 


\title{
Caractérisation microstructurale 3D et densification locale d'isolants fibreux cellulosiques sollicités en compression
}

\author{
Christine Delisée* — Eric Badel** — Jérôme Lux*** \\ Jérôme Malvestio* \\ * Unité Sciences du Bois et des Biopolymères US2B \\ UMR5103, 69, route d'Arcachon, F-33612 Cestas cedex \\ delisee@us2b.pierroton.inra.fr \\ ** INRA, UMR PIAF, 234, av. du Brezet, F-63100 Clermont-Ferrand cedex 01 \\ *** Laboratoire d'Etudes des Phénomènes de Transfert Appliqués LEPTIAB \\ EA2119, Avenue Michel Crépeau, F-17042 La Rochelle cedex 1
}

RÉSUMÉ. On s'intéresse à des isolants thermiques à base de fibres de bois utilisés dans le bâtiment, de très forte porosité, élaborés par un procédé textile non tissé. Les propriétés thermiques ont fait l'objet des premières études et les propriétés mécaniques, intervenant lors du transport et de la manipulation, font l'objet de ce travail. Nous avons suivi in situ, grâce à la microtomographie $X$, les états de déformation interne d'un panneau d'isolation soumis à une sollicitation de compression transverse (33\% et $73 \%$ ). Le comportement particulier mis en lumière par l'accès à la déformation locale est relié à la très faible densité du matériau. En particulier, les fibres de bois ne sont pas individuellement mécaniquement comprimées et la déformation macroscopique du matériau n'engendre que leur réorganisation spatiale. Ces résultats sont mis en relation avec des caractéristiques morphologiques de structures évaluées par analyse d'image $3 D$.

ABSTRACT. In this work, we focus on a thermal insulating panel made of wood fibres. The making process is a non-woven fabric process that generates a high porosity and a very lowdensity material. Thermal properties were first investigated. This paper deals with mechanical properties that are involved in the production line, transport and handling in the construction site. An insulating panel was compressed (33\% and 73\%) and we followed in situ its internal deformation using X-ray microtomography. The results enlighten a special behaviour that is linked to the low-density structure of the panel. In particular, the fibres are not compressed and the macroscopic deformation of the sample generates only a new organisation of the fibres in the panel. These results are confirmed by morphological measurements that were performed by $3 D$ image analysis.

MOTS-CLÉS : bois, compression, densification locale, fibres, isolation thermique, microstructure, microtomographie rayons $X$, morphologie.

KEYWORDS: compression, fibres, local densification, microstructure, morphology, thermal insulation, wood, X-ray microtomography. 


\section{Introduction}

L'utilisation des fibres végétales dans les matériaux destinés à l'isolation thermoacoustique des bâtiments suscite un intérêt croissant dans une démarche de qualité environnementale (label HQE). Leur utilisation reste encore cependant marginale et c'est par l'innovation que l'on pourra accroître les parts de marché de ces matériaux. La maîtrise de leur architecture constitue un point d'entrée essentiel, dans la mesure où un grand nombre de propriétés (de transport, mais aussi mécaniques, acoustiques ou optiques) sont en particulier contrôlées par la microstructure (Lux et al., 2006 ; Faessel et al., 2005). En fonction des domaines d'applications de ces matériaux (isolation thermique ou acoustique, panneau d'agencement...), on rencontre classiquement deux types de procédés d'élaboration : l'un s'appuie plutôt sur des techniques inspirées de l'industrie papetière, l'autre constitue un héritage direct de l'industrie du panneau à base de bois (procédé par thermo-compression de mats de fibres préencollées). Bien que ces techniques d'élaboration soient fondamentalement différentes (nature des liaisons, dimensions des fibres...), la principale limitation technologique reste la même; à savoir, les réseaux fibreux constitutifs ne présentent jamais une réelle architecture 3D. Plusieurs conséquences directes en découlent : la cohésion interne des panneaux demeure principalement liée à leur densité apparente et leur épaisseur se trouve de fait limitée. Ainsi, dans un contexte où l'industrie cherche à diversifier les potentialités d'applications de ces matériaux, l'élaboration de réseaux fibreux présentant une réelle architecture 3D se dégage comme l'un des axes de recherche porteurs.

Le matériau étudié est un prototype réalisé dans le cadre d'un projet industriel soutenu par l'ADEME et l'industriel ISOROY. Il s'agit d'un matériau destiné à l'isolation thermique sous toiture, de très forte porosité ( > à $95 \%$ ), constitué de $75 \%$ en masse de fibres de pin maritime et de $25 \%$ de fibres textiles bicomposantes (gaine polyester PES et âme CoPolyester Co-PES). Celles-ci assurent la cohésion (rôle de la gaine PES dont la fusion, lors du chauffage, permet d'établir les liaisons entre les fibres) tout en garantissant la tenue mécanique du matériau (rôle de l'âme Co-PES). L'objectif est d'étudier la faisabilité d'isolants naturels issus de la ressource locale (fibres de pin maritime), présentant une structure 3D fortement enchevêtrée et pouvant ainsi atteindre de fortes épaisseurs (épaisseurs $>6 \mathrm{~cm}$ ) sans contrecollage. L'architecture complexe et anisotrope du réseau des fibres (figure 1) est liée au procédé d'élaboration; ici un procédé textile non tissé. Lors d'études préalables (Lux et al., 2006; Faessel et al., 2005), nous nous sommes intéressés aux relations entre microstructure et propriétés thermiques du matériau, dans un souci d'optimisation des architectures des réseaux fibres/pores induites par les procédés d'élaboration. L'étude présentée ici entre dans le cadre d'une démarche d'écoconception des matériaux, qui consiste à optimiser les solutions techniques, industrielles mais aussi logistiques. Ainsi, après fabrication, il s'agit de conserver la qualité du produit jusqu'à et pendant son utilisation en service. Pour cela, il est nécessaire d'avoir une connaissance du comportement du matériau sous des sollicitations mécaniques, de compression en particulier, qui pourraient avoir des conséquences sur la microstructure et donc sur les 
propriétés physiques ou l'autoportance. En effet, lors des phases de stockage, de transport, de mise en œuvre sur le chantier et d'utilisation en service, ces matériaux, de forte porosité, sont soumis à des efforts de compression qui s'exercent de manière temporaire ou permanente, pouvant induire des modifications non réversibles de la structure. Par exemple, la connaissance des états de déformation interne doit être maitrisée pour envisager, sans dégradation des structures, une optimisation des masses et des volumes lors du transport et du stockage, comme c'est le cas pour d'autres types d'isolants fibreux. L'étude de la capacité de recouvrance (reprise en épaisseur) est en particulier très importante.

Dans ce cadre, un travail exploratoire de suivi des déformations sous compression, en relation avec les modifications de structure des réseaux et des fibres, est réalisé ici à partir d'images 3D acquises par microtomographie $\mathrm{X}$ à l'ESRF (European Synchrotron Radiation Facility, Grenoble) à différents taux de compression. Les essais mécaniques ont été réalisés à l'aide d'un dispositif de traction-compression spécifiquement développé au laboratoire GEMPPM (Buffière et al., 1999) pour permettre l'acquisition de scans tomographiques. Des outils issus de la morphologie mathématique (Matheron, 1967 ; Serra, 1982) sont utilisés pour traiter les images en niveaux de gris obtenues et en extraire des paramètres morphologiques et topologiques en 3D. Afin d'identifier le processus local de compression du matériau, le concept de base que nous avons choisi d'adopter pour ce matériau discontinu est celui de la conservation de la masse (Badel et al., 2003 ; Badel et al., 2008). Les résultats obtenus sur la déformation sont mis en correspondance avec le protocole expérimental et les analyses de structure réalisées par analyse d'images; permettant de mieux comprendre le processus de réorganisation des fibres au sein du matériau en liaison avec sa structure initiale.

\section{Mesures expérimentales}

Les essais mécaniques ont été réalisés sous imagerie $X$ (figure 1) sur la ligne ID19 de l'ESRF. L'échantillon testé est un cylindre de diamètre et hauteur initiale égaux à environ $10 \mathrm{~mm}$. Il a été découpé sur une des faces du panneau (d'épaisseur totale $60 \mathrm{~mm}$ ) et placé dans un tube de PMMA afin de garantir un déplacement macroscopique 1D. L'échantillon est comprimé par l'intermédiaire de supports en bois (figure 2) qui permettent de conserver, au cours de l'acquisition, une bonne dynamique des niveaux de gris. Des scans tomographiques ont été réalisés, avec une résolution spatiale de $4,91 \mu \mathrm{m}$, à trois états de compression, appelés état initial, état comprimé 1 et état comprimé 2 (figure 2). Les déformations macroscopiques expérimentales aux deux états comprimés sont respectivement estimées à 32,8\% et $72,5 \%$ par le déplacement du piston de compression. Des outils issus de la morphologie mathématique sont ensuite utilisés pour traiter les images en niveaux de gris obtenues et en extraire des paramètres morphologiques 3D (Matheron, 1967 ; Coster et al., 1985). 


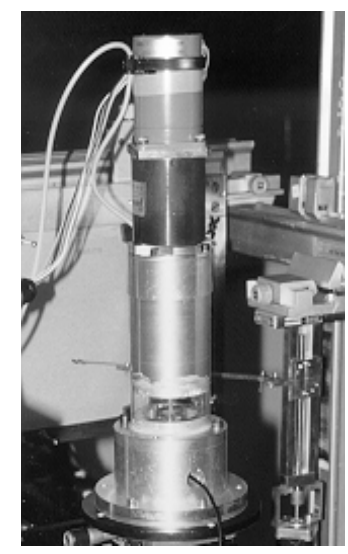

Figure 1. Essais de compression sous imagerie $X$
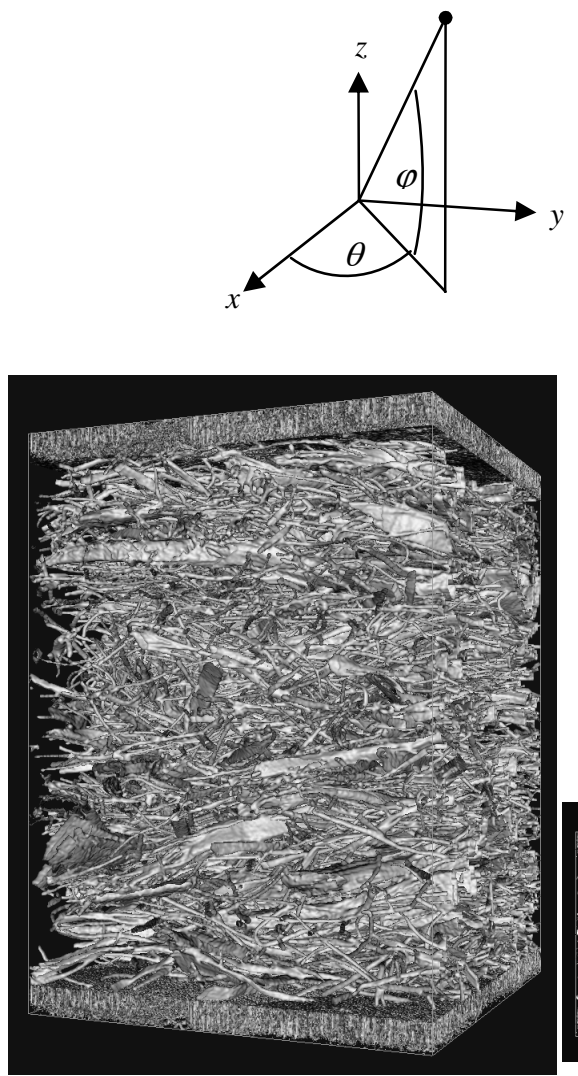

a)

b)

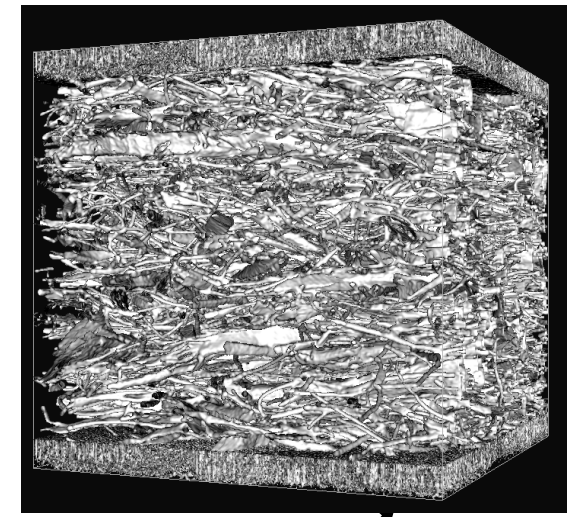

supports en bois

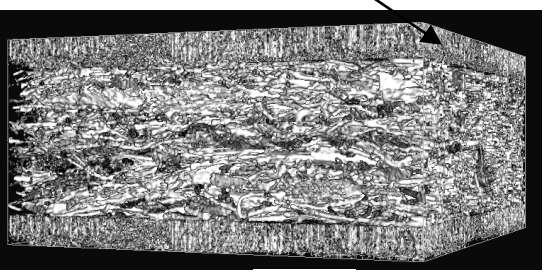

c)

Figure 2. Rendus volumiques d'un sous-volume de l'échantillon, de section $5 \times 5 \mathrm{~m}^{2}$; a) aux états initial), b) comprimé 1 et c) comprimé 2 


\section{Caractérisation morphologique 3D aux différents taux de compression}

Pour éviter les effets de bord liés à la découpe du matériau, des volumes de dimensions réduites sont extraits dans le cœur des images (figure 3). Les hauteurs des parallélépipèdes ainsi définis sont égales aux distances entre les supports haut et bas de la machine de compression. Les volumes traités ont finalement pour dimensions $1024^{2} \times 1244$ pixels $^{3}\left(5^{2} \times 6,1 \mathrm{~mm}^{3}\right)$ pour l'état initial et $1024^{2} \times 944$ pixels $^{3}$ $\left.5^{2} \times 4,1 \mathrm{~mm}^{3}\right)$ et $1024^{2} \times 342$ pixels $^{3}\left(5^{2} \times 1,7 \mathrm{~mm}^{3}\right)$ pour les deux états comprimés (figure 2). Ces dimensions sont supérieures à celles d'un volume élémentaire représentatif du matériau (VER), estimées à $\left(3,8^{2} \times 0,9 \mathrm{~mm}^{3}\right)$ pour certaines propriétés (porosités, taille des fibres et des pores, orientations) dans le cadre de travaux antérieurs (Lux, 2005) Le volume étudié ici correspond à plus de 10 fois le VER. Après filtrage et nettoyage des images pour en éliminer le bruit, les phases fibreuse et poreuse sont séparées par seuillage (figure 4). Afin de mettre en évidence les modifications structurales induites par une compression du matelas fibreux, des mesures morphologiques sont réalisées aux différents taux de compression: porosités, orientations globale et locales, dimensions des pores et des fibres.

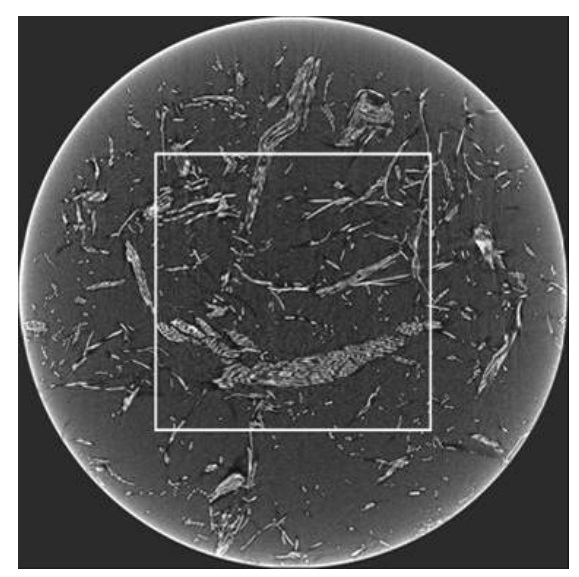

Figure 3. Coupe en niveaux de gris, dans un plan parallèle aux faces, de l'échantillon à l'état initial. Taille de l'image avant recadrage $2048 \times 2048$ pixels $^{2}$ $\left(10 \times 10 \mathrm{~mm}^{2}\right)$ et après recadrage $1024 \times 1024$ pixels $^{2}\left(5 \times 5 \mathrm{~mm}^{2}\right)$

\subsection{Porosités}

On s'intéresse, dans le cas des matériaux à base de fibres de bois, à plusieurs types de porosités. La porosité totale du matériau est obtenue à partir de l'image binaire nettoyée, en calculant la fraction volumique de l'air contenu dans un volume donné. Afin d'accéder à la porosité interne des fibres de bois (lumen), connectée à l'espace poral interfibres, des opérations morphologiques 3D (Lux et al., 2006) sont 
mises en œuvre afin de reboucher les fibres tout en préservant leurs contours (figure 4). La porosité interne est alors obtenue en ramenant le volume des lumens (volume rebouché) au volume total des fibres rebouchées. La porosité externe, ou fraction volumique des pores externes aux fibres rebouchées contenus dans un volume donné, est mesurée également. Les valeurs des porosités totale, externe et interne (des fibres) sont présentées dans le tableau 1. On constate que les porosités totale et externe sont fortement réduites dans la compression alors que la porosité interne reste quasi constante $(37,1 \%$ à l'état initial et $37,4 \%$ et $40,8 \%$ aux états comprimés). Cela indique que la taille des fibres et des bûchettes de bois n'est pas affectée par la sollicitation de compression. La valeur légèrement plus forte à l'état comprimé $2(40,8 \%)$ est probablement un artefact de mesure qui peut s'expliquer par la prise en compte, dans la porosité interne, de petits pores externes entre les fibres, crées par la densification du matériau. Ceci peut être visualisé à partir de coupes transverses du matériau avant et après rebouchage (figure 5), qui permettent de suivre l'évolution de la morphologie des fibres aux différents taux de compression. Ainsi, dans l'état comprimé 2, on observe que l'opération de rebouchage des pores internes est étendue à de petits pores externes induits par la densification.

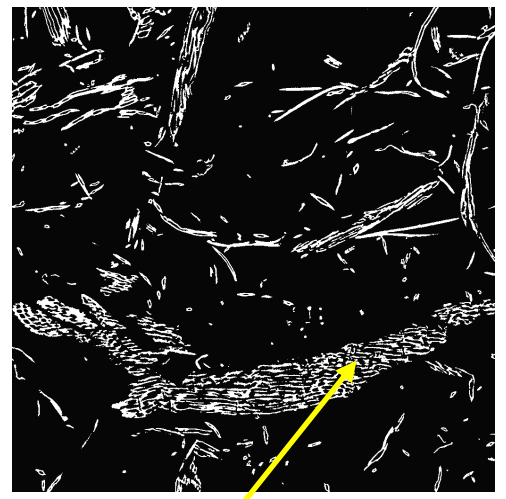

a) porosité interne des fibres

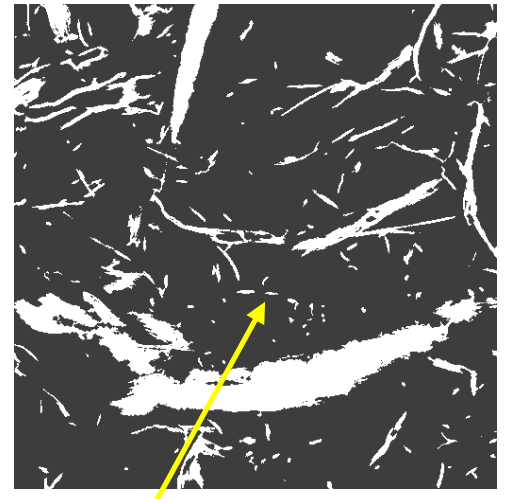

b) porosité externe aux fibres

Figure 4. Images segmentées d'une coupe (transversale à la direction de compression) : a) avant rebouchage et b) après rebouchage des fibres. Dimensions $5 \times 5 \mathrm{~mm}^{2}$

\begin{tabular}{|c|c|c|c|}
\hline Etat de compression & Porosité totale & $\begin{array}{c}\text { Porosité interne } \\
\text { des fibres }\end{array}$ & $\begin{array}{c}\text { Porosité externe } \\
\text { aux fibres }\end{array}$ \\
\hline Etat initial & $92,4 \%$ & $37,1 \%$ & $87,8 \%$ \\
\hline Etat comprimé $1(32,8 \%)$ & $88,6 \%$ & $37,4 \%$ & $81,8 \%$ \\
\hline Etat comprimé $2(72,5 \%)$ & $73,1 \%$ & $40,8 \%$ & $54,6 \%$ \\
\hline
\end{tabular}

Tableau 1. Valeurs des porosités aux 3 taux de compression 


\subsection{Distributions des tailles des pores et des fibres}

Afin d'accéder aux tailles des pores et des fibres aux deux taux de compression, on réalise respectivement sur les ensembles des pores, puis des fibres après rebouchage, des ouvertures morphologiques $3 D$ (Matheron, 1967) par un octaèdre de taille croissante (figure 6). Les courbes granulométriques obtenues montrent que, si la taille des fibres reste constante (c'est donc que les petits pores internes ne sont pas modifiés), les distances interfibres (ou taille des gros pores autour des fibres rebouchées) sont réduites lors de la compression. Toutefois, si l'on considère l'état comprimé 2 , on constate que les diamètres de fibres mesurés sont plus importants globalement et en moyenne (diamètres moyens égaux à $166 \mu \mathrm{m}$ pour l'état comprimé 2 , à $109 \mu \mathrm{m}$ et $113 \mu \mathrm{m}$ dans les états initial et comprimé 1 ). On retrouve ici les résultats observés précédemment, confirmant les limites de nos mesures dans le cas de matériaux plus denses où l'on fait apparaître des regroupements de fibres.

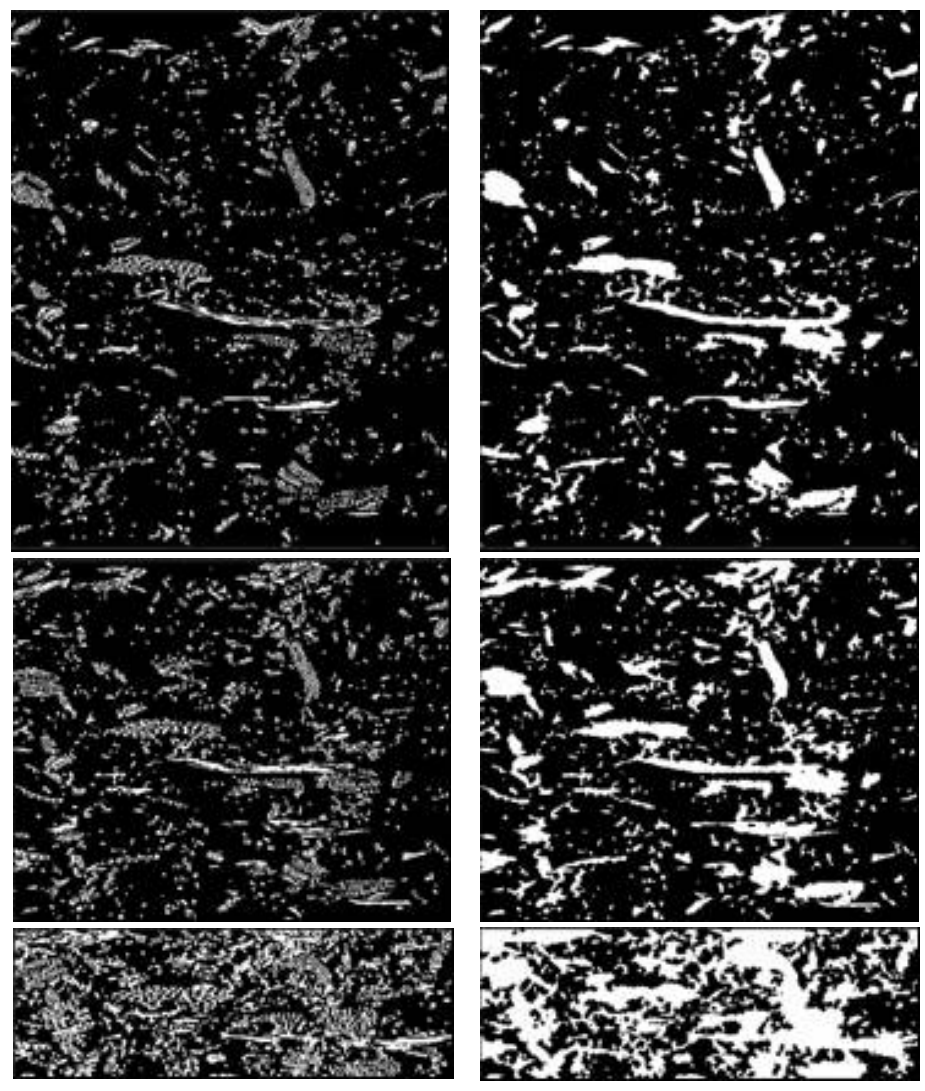

a)

b)

c)

Figure 5. Coupes transverses, avant et après rebouchage, sous la sollicitation de compression : a) état initial, b) et c) états comprimés 1 et 2 


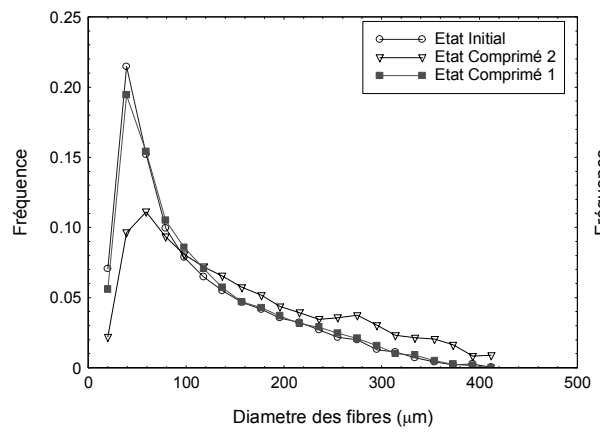

a)

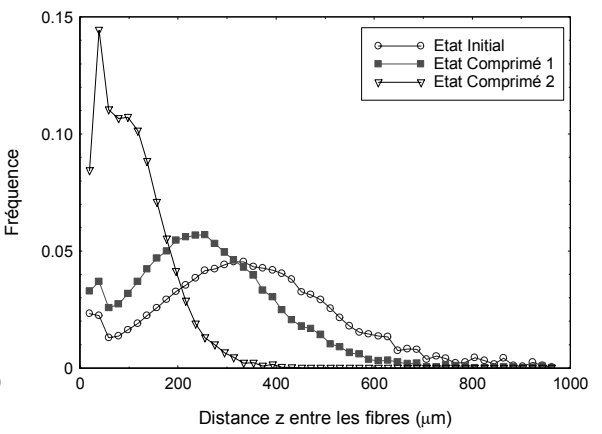

b)

Figure 6. Distributions: a) des tailles des fibres et b) des tailles des pores (ou distances interfibres)

\subsection{Anisotropie du matériau}

Le calcul des fonctions d'autocorrélation permet de mettre en évidence l'anisotropie de la structure du matelas fibreux et son évolution aux différents taux de compression. Soit $x$ et $x+h$ deux pixels (ou voxels en 3D), distants de $h$, appartenant à l'ensemble $X$ des fibres; l'autocovariance $C(h, \alpha)$ de l'ensemble $X$ des fibres rapporté au masque $Z$ (image totale) est donnée par :

$$
C(h, \alpha)=P\left\{x \in X ; x+h_{\alpha} \in X\right\}-X_{V}{ }^{2}=\frac{V\left(X \cap X_{h_{\alpha}}\right)}{V\left(Z \cap Z_{h_{\alpha}}\right)}-X_{V}{ }^{2}
$$

avec $\alpha=(\theta, \varphi)$ l'orientation du bipoint $(x, x+h)$ définie selon la figure 2, $V(X)$ le volume de $X$ et $X_{V}$ la fraction volumique des fibres.

L'autocovariance normalisée est obtenue à partir de [1] en divisant par la variance $C(0)=X_{V}-X_{V}^{2}$. Afin de limiter les modifications engendrées par la fermeture des lumens (notamment au plus fort taux de compression), nos mesures sont réalisées sur les images non rebouchées. Pour plus de visibilité, seules certaines directions, parmi les 13 directions sans biais de la trame cubique testées, sont présentées ici dans le cas de l'état initial. Les courbes obtenues dans les 4 directions parallèles aux faces sont confondues et la courbe correspondant à la direction parallèle à l'axe de compression est située très en dessous de ces courbes (figure 7), montrant que les fibres ont une orientation privilégiée dans des plans perpendiculaires à l'axe de compression et sont orientées de manière aléatoire dans ces mêmes plans. Cette anisotropie se retrouve dans tous les états de compression, même si elle est moins marquée lorsque le taux de compression augmente. Ceci résulte de l'augmentation de la fraction volumique des fibres lors de la compression. Comme le montre la figure 5, les fibres agglomérées peuvent en effet former des structures orientées dans la direction de compression. On note également 
qualitativement la diminution des longueurs de corrélation, indiquant logiquement une diminution du volume représentatif, avec l'augmentation du taux compression.

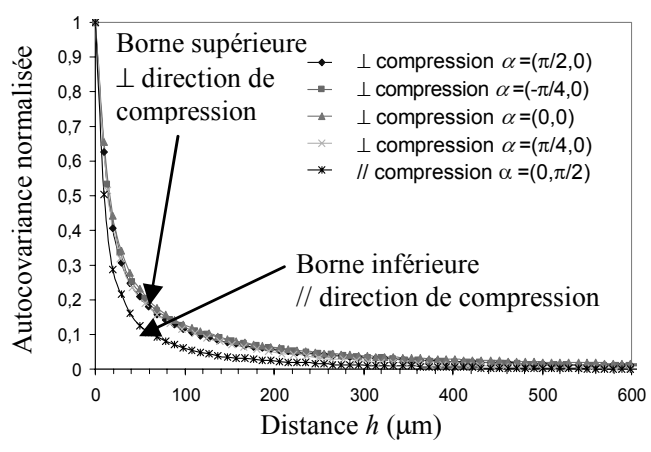

a)

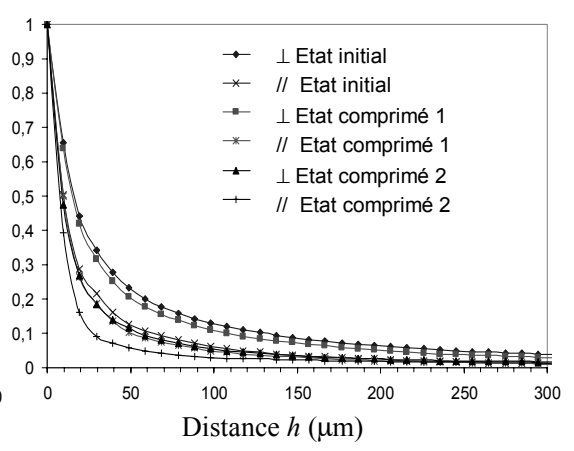

b)

Figure 7. a) fonctions d'autocovariances normalisées dans les directions longitudinales et transverses (respectivement $\perp$ et // à la direction de compression), mesurées pour l'état initial de compression; b) comparaison des courbes entre les trois états de compression

\subsection{Orientations locales}

Une définition de l'orientation locale a été initialement proposée (Soillé et al., 1998) pour l'analyse d'images en niveaux de gris. L'orientation d'un pixel $x$, appartenant à une structure plus claire que le fond, est définie par la direction $\alpha$ du segment $L_{\lambda, \alpha}$, de longueur donnée $\lambda$, qui minimise, en ce point, la différence entre le niveau de gris dans l'image originale et celui dans l'image ouverte par $L_{\lambda, \alpha}$. Cette définition a été étendue à des images binaires, présentant des structures de tailles très différentes (Lux, 2005), en utilisant des segments de longueur variable. Considérant dans un premier temps des ouvertures par des segments de longueur croissante, l'orientation d'un voxel $x$ est définie égale à celle du plus long segment inclus dans l'objet et qui passe par ce point, reflétant ainsi une propriété directionnelle plus «globale» de l'objet et non une propriété locale. Pour retrouver une définition conservant un caractère local, l'ouverture peut être remplacée par une simple érosion. L'orientation d'un voxel $x$ est alors égale à celle du plus grand segment inclus dans l'objet, qui passe par ce point et est centré sur ce point. Les deux différentes approches sont illustrées dans la figure 8 (Lux et al., 2006). L'orientation locale par érosion $D_{\lambda}(f)(x)$, relative à une longueur $\lambda$, est définie par :

$$
D_{\lambda}(f)(x)=\left\{\begin{array}{l}
\alpha_{i} \text { si } \varepsilon_{\lambda, \alpha_{i}}(f)(x)>\varepsilon_{\lambda, \alpha_{j}}(f)(x), \forall i \neq j \\
\varnothing \text { sinon }
\end{array}\right.
$$


où $f$ est l'image binaire traitée, $\varepsilon_{\lambda, \alpha}$ est l'érosion par le segment $L_{\lambda, \alpha}$ et $\varepsilon_{\lambda, \alpha}(f)(x)$ est le niveau de gris du voxel $x$ dans l'image érodée de $f$ par $L_{\lambda, \alpha}$, qui prend ici la valeur 0 ou 1. L'orientation locale par érosion $D(f)(x)$ est alors :

$$
D(f)(x)=\left\{\alpha \mid D_{\lambda-1}(f)(x)=\varnothing \text { et } D_{\lambda}(f)(x)=\alpha\right\}
$$

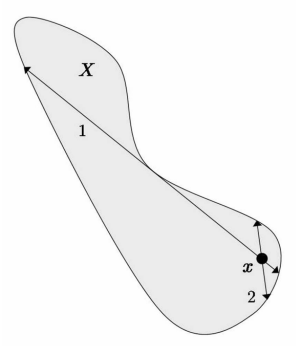

Figure 8. Représentations des orientations locales du pixel $x$ correspondant aux orientations par ouverture (segment 1) et par érosion (segment 2) (Lux et al., 2006)

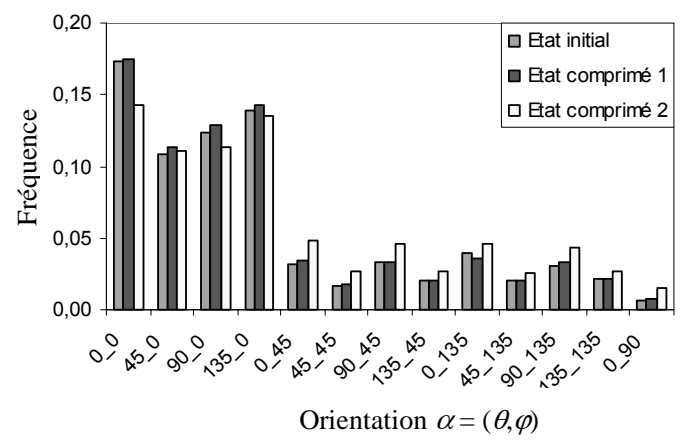

Figure 9. Distributions des orientations locales dans les 13 directions de la trame cubique

Les champs d'orientations locales ont été mesurés sur les trois images. La figure 9 présente la distribution des orientations parmi les 13 directions sans biais $\alpha=(\theta, \varphi)$ de la trame cubique. Les résultats montrent de plus que, dans le cas des états initial et comprimé 1 , plus de $70 \%$ des voxels sont orientés dans le plan xOy (plans parallèles à l'axe de compression) tandis que moins de $1 \%$ sont orientés dans la direction parallèle à l'axe Oz. Il n'y a pas toutefois de différence sensible entre ces deux états. Concernant le $2^{\mathrm{e}}$ état de compression, on constate une diminution du nombre de voxels orientés dans le plan longitudinal xOy $(63 \%)$, résultat évidemment non significatif mais prévisible d'après la figure 5 où l'on observe une 
diminution de l'anisotropie de l'ensemble global des fibres avec la compression. Ces résultats confirment l'interprétation des mesures d'autocorrélation réalisées précédemment. Dans un premier temps, la compression tend à renforcer le caractère anisotrope de la structure en orientant les fibres dans les plans xOy, puis, dans une deuxième phase, toutes les fibres entrent en contact et on tend vers une structure continue de structure apparente plus isotrope. Ceci dit, cela montre également à nouveau les limites de nos mesures basées sur une analyse globale de la phase fibreuse, dans le cas où la densification du matériau est trop importante et les fibres individuelles ne peuvent plus être identifiées. La segmentation des fibres individuelles constitue donc une étape nécessaire pour affiner la description de l'évolution du réseau fibreux.

\section{Profil des taux de compression locaux}

Afin d'identifier le processus local de compression du matériau, le concept de base que nous avons choisi d'adopter pour ce matériau est celui de la conservation de la masse (Badel et al., 2003 ; Badel et al., 2008). Nous avons émis l'hypothèse que, dans ce milieu discontinu particulier, dont l'essentiel du volume est occupé par de l'air, la déformation macroscopique de l'échantillon ne se fait, à l'échelle microscopique, que par le déplacement des points matériels constituant la phase solide du bois (parois des fibres). Ainsi, cette phase solide n'est pas comprimée mais les fibres se réorganisent simplement au sein du volume particulièrement poreux. La densité dont nous souhaitons étudier l'évolution est donc une densité de points matériels, correspondant, pour un sous-volume donné, au nombre de voxels de la phase solide. Le processus utilisé repose sur la conservation du nombre de points matériels entre l'état initial et l'état comprimé. On calcule, à partir de la tranche 1 située contre le plateau de compression, pour chaque tranche $(i)$ de l'état initial d'épaisseur 1 voxel, l'épaisseur de la tranche correspondante dans l'état comprimé ayant le même nombre de points matériels (figure 10). La déformation en compression de la tranche $(i)$ est simplement donnée par :

$$
\varepsilon(i)=\frac{e_{\text {new }}(i)-e_{\text {init }}(i)}{e_{\text {init }}(i)}
$$

avec $e_{\text {new }}$ la nouvelle épaisseur calculée dans l'échantillon comprimé et $e_{\text {init }}$ l'épaisseur initiale de la tranche dans l'état initial. Le processus est réitéré le long de l'axe de compression jusqu'au balayage complet de l'échantillon.

On obtient finalement les profils de compression locale selon l'axe perpendiculaire aux faces (figure 11). Les taux de compression macroscopiques calculés sont égaux à $32,7 \%$ et $72,4 \%$, correspondant aux valeurs expérimentales précédemment estimées. L'augmentation du taux de compression local lorsque la densité initiale diminue est mise en évidence dans la figure 12, indiquant que les particules se déplacent, sous la sollicitation de compression, préférentiellement vers les zones de faible densité. La corrélation devient très forte dans le cas d'une 
importante compression (état comprimé 2); ce qui s'explique en partie par l'homogénéisation du milieu : l'écart type sur les densités par tranche passe de $14 \%$ (état initial) à $10 \%$ (état comprimé 1) puis à $4 \%$ (état comprimé 2 ). La mise en correspondance de ces résultats avec les analyses de structure réalisées par analyse d'image permet de mieux comprendre le processus de réorganisation des fibres au sein du matériau en liaison avec sa structure initiale. En particulier, la très forte porosité et le mode de liaison des fibres jouent un rôle primordial dans ce comportement mécanique en compression.

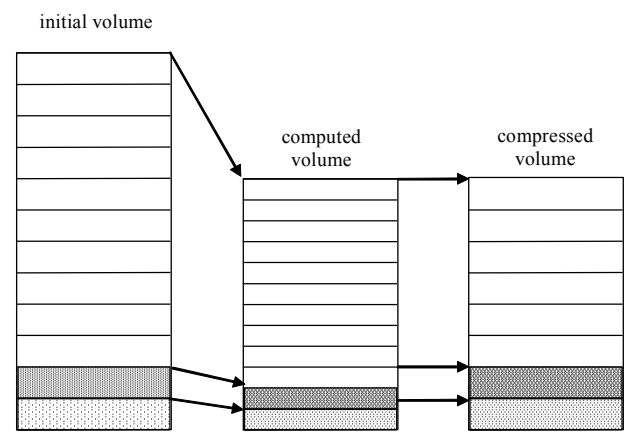

Figure 10. Processus de densification locale par tranche

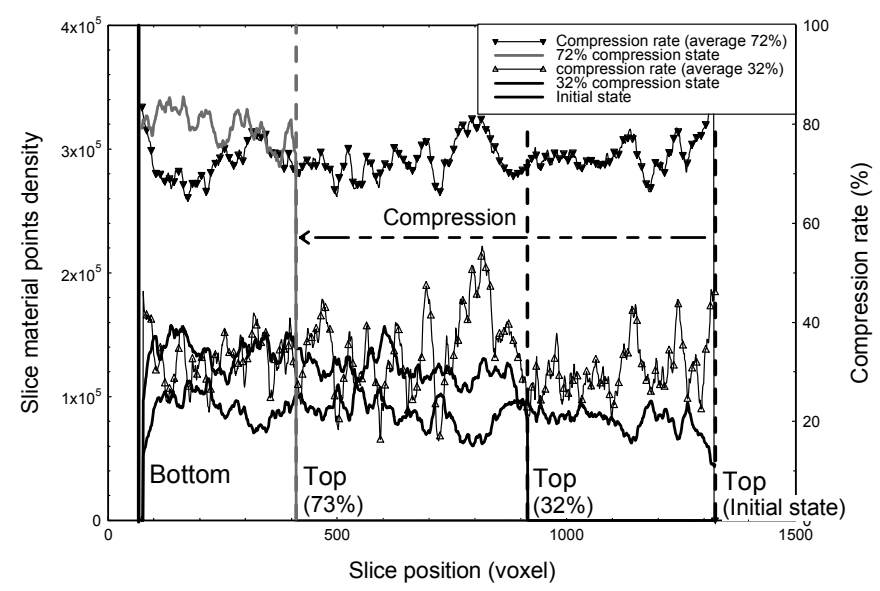

Figure 11. Profils de distribution de la matière selon l'axe de compression et profils de compression locale pour $33 \%$ et $73 \%$ de compression globale de l'échantillon 


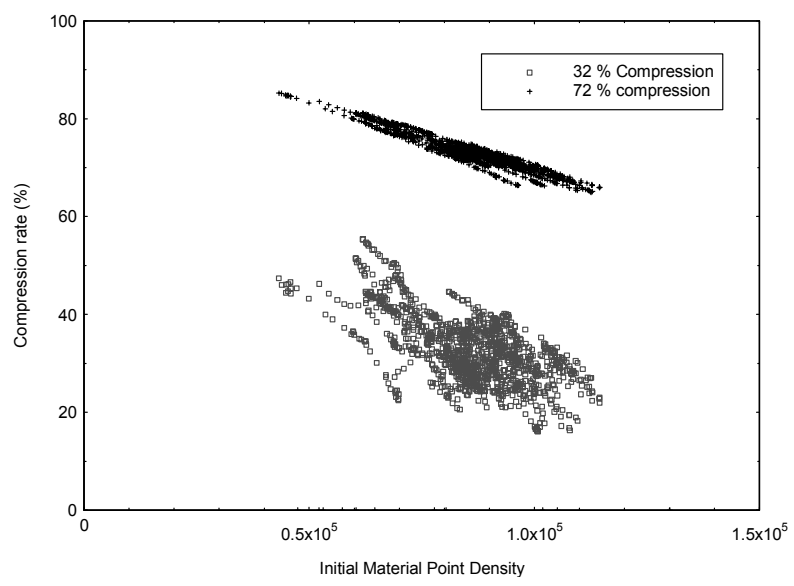

Figure 12. Relation entre la densité initiale de points matériels (intégrée par tranche transversale) et le taux de compression local

\section{Conclusion}

Des images microtomographiques de matériaux isolants à base de fibres de bois, de densité très faible, ont été réalisées à différents taux de compression, afin de mettre en évidence les modifications de structure et les déformations locales induites par la sollicitation. Le processus local de compression du matériau est identifié en utilisant le principe de conservation de la masse entre un état initial et un état comprimé, avec l'hypothèse d'un déplacement moyen des particules solides suivant l'axe de compression. De plus, dans ce milieu discontinu et essentiellement composé d'air, la déformation macroscopique ne se fait que par le déplacement des points matériels constituant la phase solide du bois, sans densification intrinsèque de la matière fibreuse. Nous avons ainsi suivi l'évolution de la densité des points matériels, correspondant, pour un sous-volume donné, au nombre de voxels de la phase solide. Ces hypothèses ont pu être validées par des mesures morphologiques réalisées sur les images : porosités, tailles des fibres et des pores et orientation. La porosité interne des fibres n'est pas affectée, contrairement à la porosité externe aux fibres. La phase fibreuse n'est donc pas comprimée; les fibres se réorganisent quasiment librement au sein du volume, et plus particulièrement dans les zones de faible densité. Les fines liaisons plastiques entre les fibres ne semblent pas avoir de rôle mécanique lors de la compression. Cela peut être interprété par un comportement de type "corde», capable d'exercer des efforts en tension, qui maintiennent la cohésion du panneau, mais pas en compression. Ainsi, le protocole d'analyse proposé, basé sur la microstructure particulière du matériau (discontinuité du matériau, très basse densité) et le protocole expérimental donnent des résultats en adéquation avec les mesures extraites des images et les mesures de déplacement 
réalisées lors de l'expérimentation. En perspectives, un traitement plus complet, de type corrélation d'images 3D, avec un suivi de point, est envisagé. De plus, des mesures morphologiques complémentaires, réalisées à partir d'une représentation simplifiée du réseau sous forme d'un squelette $3 \mathrm{D}$ et permettant de caractériser et suivre l'évolution des contacts entre les fibres (nombre, surface de contact, répartition dans l'espace), sont en cours de réalisation. Enfin, si cette étude, effectuée à l'échelle mésoscopique, reste nécessaire et doit être approfondie, une perspective à moyen terme est l'intégration des observations obtenues à cette échelle au comportement à l'échelle macroscopique par des techniques d'homogénéisation.

Les auteurs remercient l'équipe de la ligne ID19 (ESRF) et plus particulièrement Xavier Thibault pour l'aide apportée lors des phases d'expérimentation.

\section{Bibliographie}

Badel E., Delisée C., Lux J., "3D structural characterisation, deformation measurements and modelling of low-density wood fibreboard under compression. The use of microtomography", Composites Science and Technology, vol. 68, 2008, p. 1654-1663.

Badel E., Létang J.M., Babot D., "Quantitative microtomography: measurement of density distribution in glass wool and local evolution during a one-dimensional compressive load", Meas. Sci. Technol., vol. 14, 2003, p. 410-420.

Buffières J.Y., Maire E., Cloetens P., Lormand G., Fougères R., "Characterization of internal damage in a MMCp using X-ray synchrotron phase contrast microtomography", Acta Materialia, vol. 47, $\mathrm{n}^{\circ}$ 5, 1999, p. 1613-1625.

Coster M., Chermant J.-L., Précis d'analyse d'images, Presses du CNRS, 1985.

Faessel M., Delisée C., Bos F., Castéra P., "Modelling of random cellulosic fibrous networks based on X-ray tomography and image analysis", Composites Science and Technology, vol. 65, 2005, p. 1931-1940.

Lux J., Comportement thermique macroscopique de milieux fibreux anisotropes : étude basée sur l'analyse d'images tridimensionnelles, Thèse de doctorat, Université Bordeaux 1, 2005.

Lux J., Ahmadi A., Gobbé C., Delisée C., "Macroscopic thermal properties of real fibrous materials: Volume averaging method and 3D image analysis", Int. J. Heat Mass Transfer, vol. 49, 2006, p. 1958-1973.

Lux J., Delisée C., Thibault X., "3D characterization of wood based fibrous materials: an application", Image Analysis and Stereology, vol. 25, p. 25-35.

Matheron G., Eléments pour une théorie des milieux poreux, Paris, Masson, 1967.

Serra J., Image Analysis and Mathematical Morphology, New-York, Academic Press, 1982.

Soillé P., Talbot H., "Directional morphological filtering", Image Analysis and Mathematical Morphology, New-York, Academic Press, 1982. 